\title{
Coupling multi-agent and macroscopic simulators of traffic
}

\author{
Xavier Boulet ${ }^{1,2}$, Mahdi Zargayouna ${ }^{1}$, Gérard Scemama ${ }^{1}$, Fabien Leurent ${ }^{3}$ \\ ${ }^{1}$ Université Paris-Est, IFSTTAR, COSYS, GRETTIA \\ Boulevard Newton, Champs sur Marne \\ F-77447 Marne la Vallée Cedex 2, France \\ ${ }^{2}$ IRT SystemX - 8 Avenue de la Vauve, 91120 Palaiseau - France \\ ${ }^{3}$ University of Paris-Est, Ecole des Ponts Paristech, IFSTTAR, LVMT, France \\ $\{$ xavier. boulet, zargayouna, gerard.scemama\}@ifsttar.fr \\ fabien. leurent@enpc.fr
}

\begin{abstract}
Traffic simulations exist in multiple scales and each of these scales presents some advantages and is useful in certain contexts. Usually, multi-agent simulations use more detailed models and give more precise results than macroscopic models but their high calculation cost does not allow them to simulate very big areas such as an entire region. To overcome these limitations, multi-scale simulations emerged with the coupling of two or more simulations of different scales. This paper presents a generic solution to combine a macroscopic simulator working on a large area which contains a smaller area simulated by a multi-agent simulator. The main challenge is to assure the coherence between both simulators on the smallest area since it is simulated by both simulators at the same time. We first highlight the issues to tackle and the problems to solve when coupling two existing simulators then we propose solutions for the coupling, and finally evaluate them on an example scenario.
\end{abstract}

\section{Introduction}

To understand the mobility problems in a local area, such as traffic congestion or public transport overcrowding and to propose an efficient mobility policy, simulation is a necessary tool to bring the best solution before impacting the real traffic. However, simulating the only zone of interest is myopic and simulations should consider the surrounding areas, which can influence the behavior of the local area. Conversely, a policy change on the local area would have consequences on the rest on the multi-modal transportation network. In our use case, we consider an area of interest inside a large city where we desire to manage the multi-modal traffic and propose new ways of mobility which imply a change of transportation supply during the simulation. To do so, we simulate both the area of interest and the larger city at different scales at the same time in order to predict abnormal flows of travelers travelling by the area of interest especially in the case of a disruption of public transport in another part of the city. This is why we are interested in multi-scale simulations: to allow the simulation of 
different point of views of a phenomenon with at least two simulators of different scales working in interaction.

This paper is structured as follows. In section 2, we present related studies and existing software for multi-scale simulation between a macroscopic model and a multi-agent model. In section 3, we define our precise context and present a specification of the middleware that we propose. In section 4 , we present our experimental results before to conclude the paper.

\section{Related work}

There are many existing simulations combining a microscopic or multi-agent model with a macroscopic one. For instance, [1] proposes a dynamic traffic model focusing on the visualization, without bidirectional exchange between the macroscopic and the microscopic representation. It allows to zoom on any part of the macroscopic simulation and to see it with a multi-agent model. [2] instead decomposes the network into microscopic and macroscopic clusters that exchange data, these clustering can change during the simulation to follow a phenomenon like a shockwave. These two examples concern the combination of two models inside the same simulator but there are other works about coupling two different simulators. One of the first coupling between two simulators of different scales is in [3] and [4] with the microscopic traffic simulator PELOPS and the macroscopic simulator SIMONE where they have to solve problems like the difference between time steps of the simulators and how to generate the mobility behavior of travelers with the distribution of time gaps and speed fluctuations for the microscopic model. MICMAC [5] proposes the coupling of the microscopic model SITRA B+ and the macroscopic one SIMRES. Hystra [6] is focused on the coherence between both models and defines the conditions that they have to verify to be able to work together.

All of these works are focusing on two specific existing models which are used to simulate the same area. In this paper we propose to focus on the use case of a small area simulated with a multi-agent simulator inside a larger area simulated with a macroscopic model. We do not constrain both models and we try to propose a generic approach to this case to be able to use any already existing simulator (e.g. SM4T $[11,12])$. So, instead of modifying the used models to be coherent together, we insert a middleware between them that handles the exchanged data and the synchronization between simulations.

\section{Middleware for coupling traffic simulations}

\subsection{Context}

As we explained in the introduction, both simulators interact together to have every advantage of the two scales of simulation. The macroscopic simulator works on the bigger area which represents the region and the multi-agent system works on a smaller area included in the first, which represents the neighborhood. 
Coupling multi-agent and macroscopic simulators of traffic

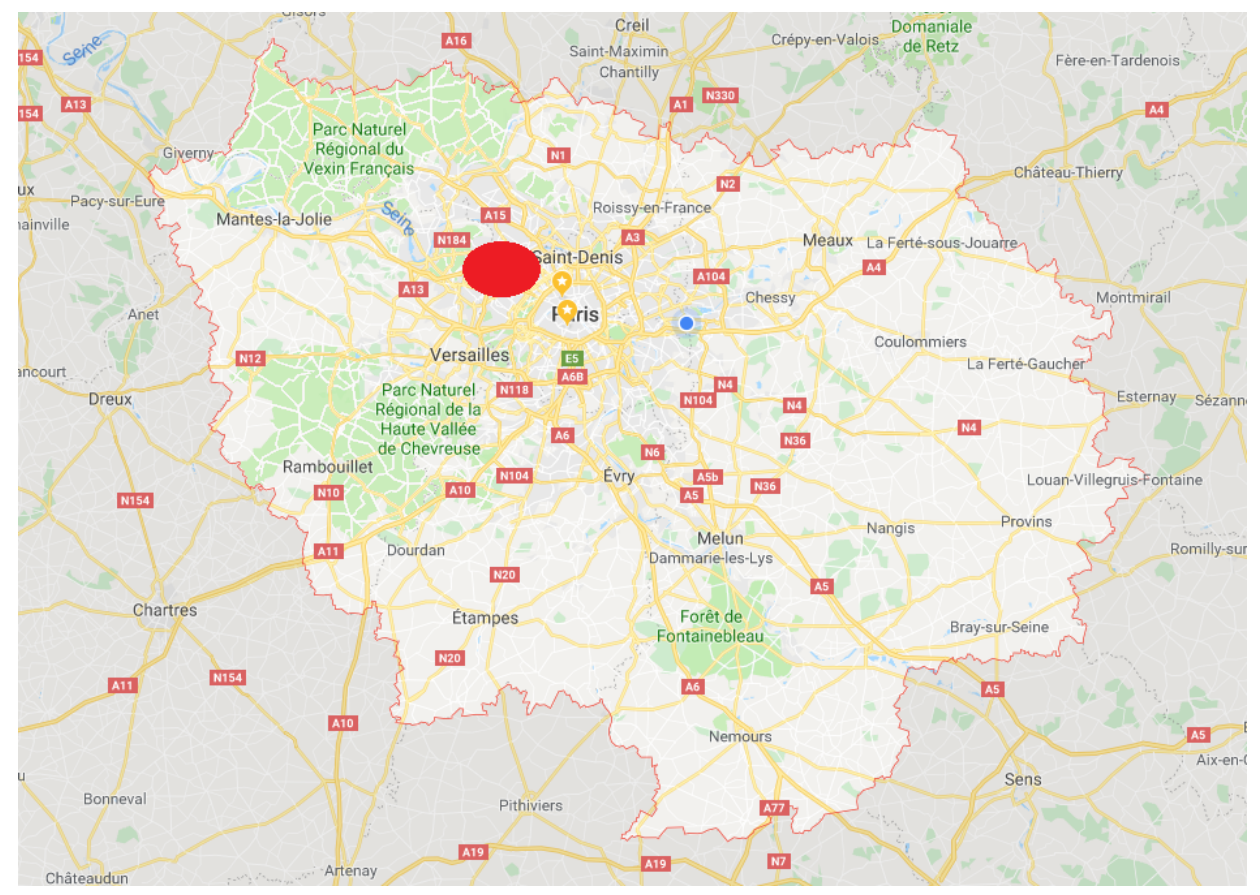

Fig. 1. Multiscale simulation of the region (in red the local simulation zone)

Both simulators can work independently, they have their own demand represented by time dependant origin-destination matrix, their own way to assign the travelers on their network and their own behavior model to calculate the speeds of their travelers.

Even if they can be executed independently, we need both simulations to be coherent when they are used together. For instance, if we observe a congested road in the macroscopic simulator, we should observe the same congestion at the same time in the local simulator. To ensure this coherence, we consider three possible corrections of the simulators:

- The correction of the demand. The demand is usually made of the origindestination matrix of the area of interest. The designer has to decide which simulation comprises the most correct information and should therefore correct the other. The regional simulator usually has a more complete view of the demand. Indeed, mobility surveys usually concern a regional scale. However, if a more precise study of the demand has been made for the area of interest for the local simulator, then the local simulation should correct the macroscopic simulation.

- The correction of the travellers' itineraries. Travelers itineraries are the result of the assignment model of the considered simulation. Simulations might not 
use the same assignment mode, and the designer has to define which one should correct the other.

- The correction to the travellers' movements. Travelers' movements define their speeds and travel times. The simulators have possibly different models for travelers movements, the designer has to decide which model should correct the other.

These three corrections are necessary to be able to observe the same phenomena of congestion, delay, path changing, etc. in both simulators.

The first step is then to chose which simulator will correct what. If only one simulator corrects everything then we don't need two simulators in interaction, the correcting one can be used alone then his results can be used by the second simulator that will be run alone in a second time. In our use case, we consider that the macroscopic simulator is used to bring a demand that has already been assigned to the multi-agent simulator and the latest will correct the behavior of the travelers.

\subsection{The middleware}

The three types of scale that we consider for the middleware are: the entities representation, the spatial representation and the time step.

- The representation of mobile entities: The macroscopic models work with flows of travelers whereas the multi-agent simulator represents travelers individually.

- The spatial representation: Macroscopic simulators represents the transport network in a simplified way. The network is often aggregated and some links can be deleted to optimize computational cost [7]. The more detailed models use bi-dimensional layers with grid cellular automata model [9] or continuous space [8].

- The time step: A regional assignment works with aggregated period of time (15 mn, $30 \mathrm{mn}, 60 \mathrm{mn}$, etc.) while a microscopic simulation usually considers a few seconds time steps.

In our case we consider that the macroscopic simulator works with flows on travelers, on an aggregated network and a timestep of $10 \mathrm{mn}$. The multi-agent simulator works with agents representing individual travelers, on a more detailed network (more roads and intersections) and with a timestep of $1 \mathrm{mn}$.

We consider that the multi-agent simulation corrects travelers' movements while the macroscopic simulation corrects the demand and the travelers' assignments.

The middleware has to manage the correction while coordinating the two simulators with different time steps, diffrent entities representation and different movement model. 
Coupling multi-agent and macroscopic simulators of traffic

\subsection{Synchronising the simulators}

The middleware becomes the scheduler of the multiscale simulation. It launches simulator to execute a time step, retrieves the dynamic simulation data and can block both simulations until the data that they need are available.

At each time step, the multi-agent simulator has to be fed with the demand and the assignment from the macroscopic simulator, and at each time step the macroscopic simulator has to been fed with the links speed of the multi-agent simulator.

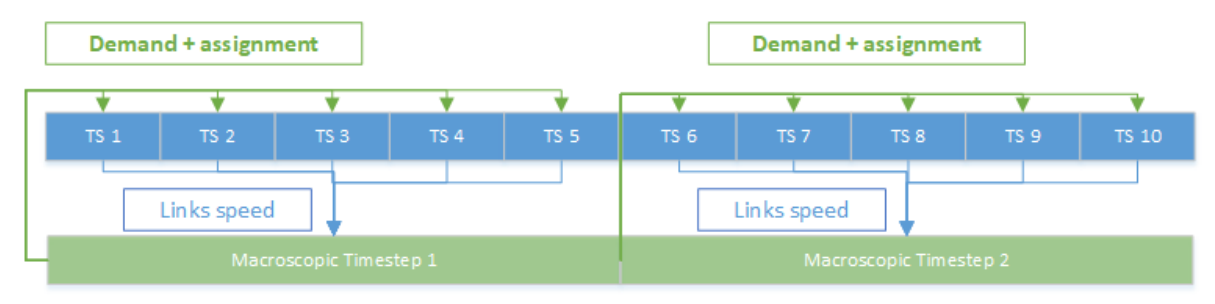

Fig. 2. Parallel execution of two simulators

At this point, the designer faces an deadlock situation: each simulator needs the other to be executed first to be corrected. We have two choices:

- We either correct the demand and the assignment for time step 1 (TS1), then we execute (TS1,..,TS5) and we will be able to bring the average of the movement behavior calculated during (TS1,..,TS5) and feed it to the macroscopic simulator.

- Or we correct the demand and the assignment for TS1, we execute TS1 and we use the movement behavior calculated in TS1 to correct the macroscopic timestep 1, then we run the macroscopic timestep 1 and we will be able to correct the demand and the assignment for every multi-agent simulation time step (TS1,..,TS5)

In the first case, the multi-agent simulator does not receive the travelers from the macroscopic simulator until TS6 and instead of having a small input on every time step, we will have a huge input every 5 time steps so the correction of the demand will be incorrect. In the second case, the macroscopic time step 1 will only be corrected with the links speed from TS1, so if there is a huge congestion at TS2 it will not be taken in account in the macroscopic simulator until his second time step so the correction of the behavior will be incorrect.

Our proposed solution is to introduce a rollback for the macroscopic simulator:

- We correct the demand and the assignment for TS1 and run TS1.

- We correct the movement behavior for the macroscopic timestep 1 and we run it 
- We correct the demand and the assignment for TS2 and run TS2 thanks to macroscopic results

- We correct the macroscopic timestep 1 with average speed of TS1 and TS2

- etc.

\subsection{Transformation between agents and flows}

In this part, we focus on the demand correction between both simulators. Whenever travellers in the macroscopic simulator enter in the area of interest, they need to be created in the multi-agent simulator but we have two different representations of our travelers since there are flows in the macroscopic model and we want new agents.

[1] presents how to change the traveller's representation between flows and agents dynamically inside a single simulator. Even if this is not our use case the same transformations can be made between two simulators to obtain the right representation of the travellers. The easiest part is the transformation from agents to flows since it corresponds to the calculation of the average density and speed. If the aggregation of information is straightforward with averaging functions, the reciprocal transformation, from flows to agents, needs the creation of new information. [1] presents a method based on gaz cinetic theory. It consists of creating agents at position in a probabilistic way. So when travelers enter the area of interest, they can be created and divided on their link of arrival.

\subsection{Correction of assignment and behavior on different network representation}

Our last focus is on the difference between the networks used by both simulators. When the simulated area is large enough, the network can be aggregated in order to speed up the simulation [10]

Figure 3 shows an example of a network with an aggregated representation (left) and a detailed one (right). We consider that every node of the aggregated representation can be found in the detailed one since a node usually represents an existing physical place and if this place is present in the macroscopic simulator, it will necessarily be in the most detailed one too. This is not the case for the links since in the aggregation process new links can be created between nodes even if these links are not existent physical roads, they can be the aggregation of some existing links. Our goal is to determine how to correct the assignment and the movement behavior despite this difference.

For the assignment, the first step is to find for every link in the macroscopic network, every path corresponding in the detailed network. For every couple $\left(S_{i}, S_{j}\right)$ in the macroscopic network, we have to find every path with $S_{i}$ as origin and $S_{j}$ as destination such as no other macroscopic node belongs to this path. Here is an example in the following figure: The link $\left(S_{2}, S_{4}\right)$ corresponds to the paths $\left(S_{2}, s_{5}, S_{4}\right)$ and $\left(S_{2}, s_{6}, S_{4}\right)$. 
Coupling multi-agent and macroscopic simulators of traffic
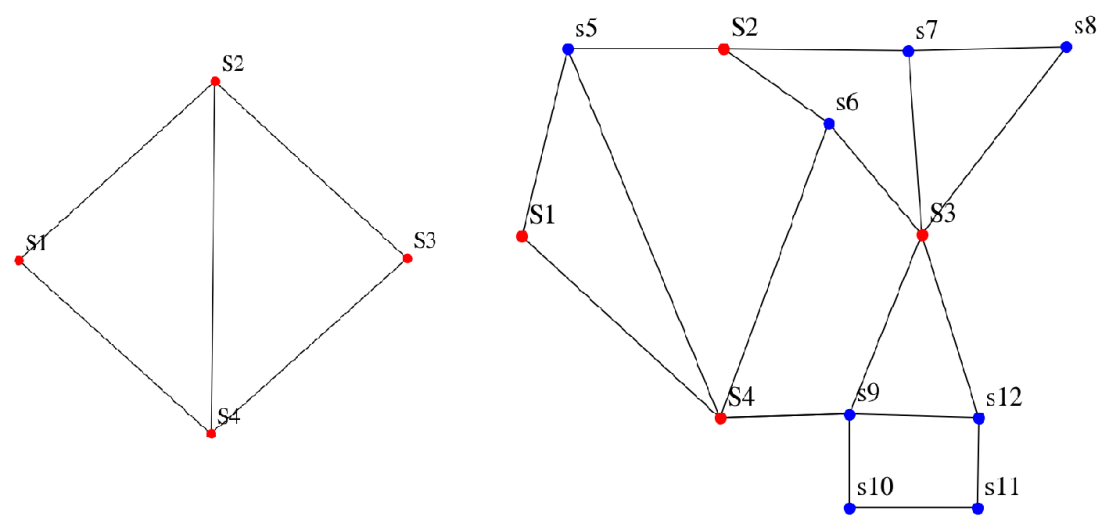

Fig. 3. Two different representations of the same network
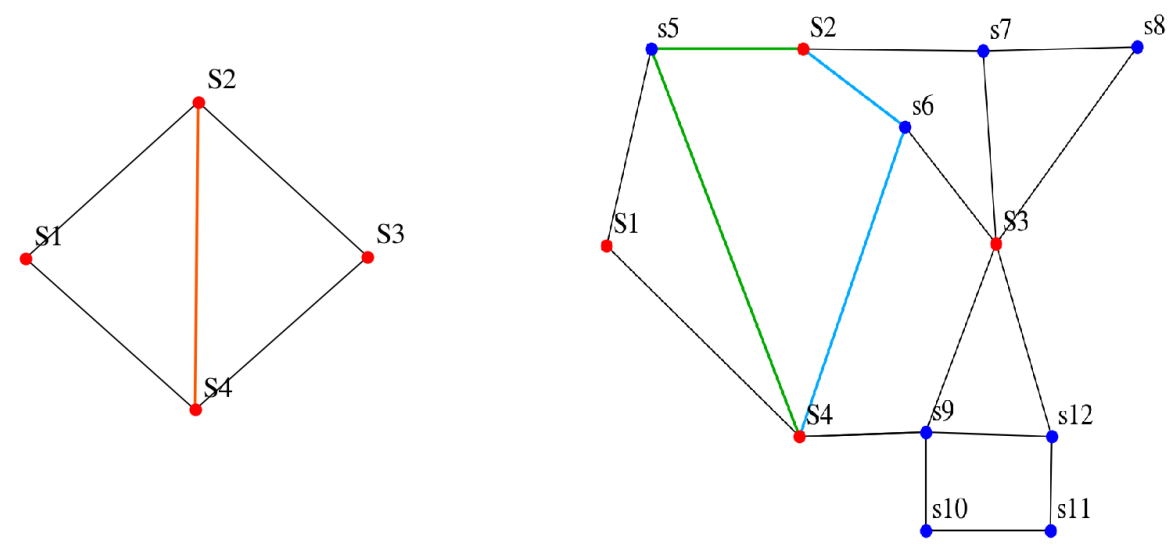

Fig. 4. Highlight of the paths corresponding to a macroscopic link

Then we know that every traveler passing by the link $\left(S_{2}, S_{4}\right)$ will pass on one of the two corresponding path. From there, the assignment model of the multi-agent simulator will determine which path they have to take and it will assign them. So the correction of the assignment from the macroscopic simulator to the multi-agent simulator is, in fact, an assignment made by the multi-agent simulator constrained by the assignment of the macroscopic one.

Finally, we need to correct the movement behavior from the multi-agent simulator to the macroscopic one. To do so we will once again use the macroscopic 
Xavier Boulet, Mahdi Zargayouna, Gérard Scemama, Fabien Leurent

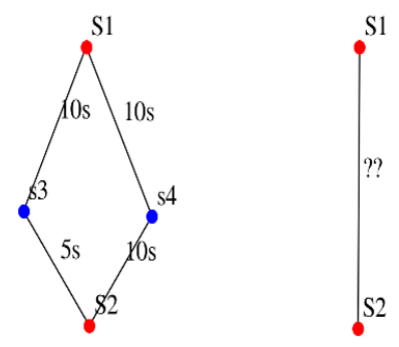

Fig. 5. Correction of the macroscopic link speed

links and their corresponding detailed path. But some incoherence are inevitable as we can see in the figure 5 .

Two paths with different time travel can be aggregated into the same macroscopic link. In this case the travelers taking these different path will have the same speed on the macroscopic simulator but not in the multi-agent simulation. We chose to give the macroscopic link a travel time corresponding the a weighted average of the microscopic path time travel, the weight being the number of travelers on each path.

\section{Experiments}

To validate the proposed middleware, we implemented two simulators and used our middleware to make them work together. The macroscopic simulator works with flows on travelers, on an aggregated network and a time step of $10 \mathrm{mn}$. The multi-agent simulator works with agents representing individual travelers, on a more detailed network and with a time step of $1 \mathrm{mn}$. The macroscopic simulator assign flows of travellers always on the shortest path from their origin to their destination based on these paths travel time during the last time step, the multi-agent simulator assigns travellers using a K-shortest path algorithm so two travellers with the same origin and destination do not always take the same path. The multi-agent simulator uses a fundamental diagram to determine the speed on the links so they are slowed down when there are too many travellers. Finally, the demand used for this simulation is almost constant and low during most of the simulation with a peak corresponding to a rush hour.

We looked at the difference of travel time during their entire journey in the area of interest between the flows of travellers in the macroscopic simulator and the traveller agents created from these flows. These agents can be as much as $10 \%$ slower or faster than the corresponding flows. This difference is minimized by our choice of correcting macroscopic links speed with a weighted average of the microscopic path time travel and obviously these numbers depend on the way the aggregated network has been constructed compared to the detailed one. 
We then focused on the peak of demand which created some congestion and slowed down many links of the area as expected. Our objective was to see if the macroscopic links slowed down by the movement correction had an increase of their number of travelers at the same time. Once again, it depends a lot on the network, but every macroscopic link slowed down by the correction saw the planned increase of travellers corresponding to the congestion, in some cases 1 or 2 time steps later.

These results suggest that the middleware is working well in this sample scenario, and performs the needed corrections between simulations running at different scales.

\section{Conclusion}

In this paper, we tackle the problem of multiscale simulation, and adopt a middleware-based solution. The idea is to reuse existent simulators and add a third part: the middleware. We have presented the problems faced during the coupling of simulations working at different scales, and provided a specification of the middleware. The experimental results on a sample scenario suggests that this solution is a relevent one, and provides the right corrections between the two simulations. In our future works, we plan to generalize the middleware model for any type of simulations, and to implement a real world middleware working on two real operational simulations.

\section{References}

1. Sewall, J., Wilkie, D. and Lin, M. C. 2011, Interactive hybrid simulation of largescale traffic, ACM Transaction on Graphics (Proceedings of SIGGRAPH Asia) 30(6)

2. Bouha, N., Morvan, G., Abouaissa, H. and Kubera, Y. 2015 A First Step Towards Dynamic Hybrid Traffic Modeling, Proceedings of 29th European Conf. on modelling and simulation (ECMS), p. 64-70

3. Lerner, G., A. Hochstaedter, R. Kates, 2000. The interplay of multiple scales in traffic flow: coupling of microscopic, mesoscopic and macroscopic simulation. World Congress on Intelligent Transportation Systems, Torino.

4. Kates, R., A. Poschinger, 2000. Investigation of a stochastic disaggregation model. World Congress on Intelligent Transportation Systems, Torino

5. Magne, L., S. Rabut, J.-F. Gabard, 2000. Towards an hybrid macro-micro traffic simulation model. INFORMS, Salt Lake City, USA

6. Bourrel, E., J.-B. Lesort, 2003. Mixing Micro and Macro Representations of Traffic Flow: a Hybrid Model Based on the LWR Theory. Transportation Research Board, Washington DC

7. R.D. Connors, D.P. Watling. Assessing the demand vulnerability of equilibrium traffic networks via network aggregation. Networks and Spatial Economics. ISSN 1566-113X, 2014

8. C.J.E. Castle, N.P. Waterson, E. Pellissier, S. Le Bail, In: Peacock R., Kuligowski E., Averill J. (eds) Pedestrian and Evacuation Dynamics. Springer, Boston, MA, 2011 
Xavier Boulet, Mahdi Zargayouna, Gérard Scemama, Fabien Leurent

9. S. Sarmady, F. Haron, A.Z.Talib. Simulation of Pedestrian Movements Using Fine Grid Cellular Automata Model.12th International Conference On Computer Modelling and Simulation, 2014

10. Paluch, S., Pesko, S., Majer, T. 2015. Transportation Network Reduction, Faculty of Management Science and Informatics, University of Zilina

11. Zargayouna, M., Othman, A., Scemama, G., \& Zeddini, B. (2015, September). Impact of travelers information level on disturbed transit networks: a multiagent simulation. In 2015 IEEE 18th International Conference on Intelligent Transportation Systems (pp. 2889-2894). IEEE.

12. Zargayouna, M., Othman, A., Scemama, G., \& Zeddini, B. (2018). Multiagent simulation of real-time passenger information on transit networks. IEEE Intelligent Transportation Systems Magazine. 